\title{
Humidity responsivity of poly(methyl methacrylate)- based optical fiber Bragg grating sensors
}

\author{
Wei Zhang* and David J. Webb \\ Aston Institute of Photonics Technology, Aston University, B4 7ET, UK \\ *Corresponding author: w.zhang@aston.ac.uk
}

Received February 10, 2014; revised April 11, 2014; accepted April 12, 2014; posted April 14, 2014 (Doc. ID 206246); published May 14, 2014

\begin{abstract}
The humidity response of poly(methyl methacrylate) (PMMA)-based optical fiber Bragg gratings (POFBGs) has been studied. The characteristic wavelength of the grating is modulated by water absorption-induced swelling and refractive index change in the fiber. This work indicates that anisotropic expansion may exist in PMMA optical fiber, reducing the humidity responsivity of the grating and introducing uncertainty in the responsivity from fiber to fiber. By pre-straining a grating, one can get rid of this uncertainty and simultaneously improve the POFBG response time. (c) 2014 Optical Society of America

OCIS codes: (060.2370) Fiber optics sensors; (060.3735) Fiber Bragg gratings; (250.5460) Polymer waveguides. http://dx.doi.org/10.1364/OL.39.003026
\end{abstract}

Bragg gratings have been successfully inscribed into poly (methyl methacrylate) (PMMA)-based plastic optical fiber in both step-index and microstructured geometries. The physical and chemical properties of polymeric materials are rather different to silica and may offer advantages in certain situations. Since polymers are biologically compatible with human tissues, PMMA optical fiber Bragg gratings (POFBGs) are particularly appealing in medical devices. The interesting features of POFBGs include the negative refractive index (RI) change against temperature rise (the opposite of that for silica gratings) and affinity for water that leads to a swelling of the fiber and an increase of RI. The former feature offers a wellconditioned performance for overcoming the cross sensitivity issues existing in silica fiber [1] , while the latter feature leads to an increase with humidity of the Bragg wavelength of a Bragg grating written in the fiber [2]. This is a potentially very useful property that has possible applications in the chemical processing, agricultural, food storage, paper manufacturing, semiconductor, and pharmaceutical industries, where humidity is monitored and controlled to ensure product quality. This property has also been exploited for the detection of saline concentration [3] and water in fuel [4]].

There have been many reports on the use of PMMAbased fiber gratings for humidity sensing. However, the reported humidity responsivities show considerable variation (see, e.g., $[\underline{1}, 2, \underline{5}])$. This variation has not been satisfactorily explained and causes great uncertainty concerning the POFBG's performance and applicability.

The Bragg wavelength of a fiber grating depends on the effective core RI and the grating pitch, both of which depend on the temperature and the water content in the case of a PMMA-based POFBG. For constant temperature, the Bragg wavelength change of a POFBG against humidity can be expressed as

$$
\Delta \lambda_{B}=\lambda_{B}(\eta+\beta) \Delta H,
$$

where $\lambda_{B}$ is the initial Bragg wavelength, $\eta$ is the normalized RI change with humidity $(\% \mathrm{RH})^{-1}$, and $\beta$ the swelling coefficient related to humidity induced volumetric change $(\% \mathrm{RH})^{-1}$. The wavelength change in POFBG sensors varies linearly with humidity, if $\eta$ and $\beta$ are constant; however both $\eta$ and $\beta$ can be temperature or humidity dependent [6,7]. It should be pointed out that not every polymer shows this water affinity: Bragg gratings made in TOPAS polymer are insensitive to humidity [] $]$ and are therefore suitable in applications where cross sensitivity to humidity needs to be avoided.

Bragg gratings can be written into pure PMMA optical fibers by using UV illumination to induce polymerization of unreacted monomers that contribute to an increase in RI. Better performance has been achieved using dopedcore PMMA-based optical fibers [9]. Since additional dopants or copolymers are required to control the core index of step index fibers, the performance of PMMAbased fiber gratings may vary with the type of fiber used. The detailed composition of dopant and copolymer used in a specific PMMA-based optical fiber is often not known and neither are their dependence on temperature and humidity. Therefore the performance of a POFBG sensor is uncertain in many cases.

From Eq. (1), one can see that there are two factors contributing to the wavelength change of a POFBG. One is the RI change induced by the humidity change, i.e., the RI humidity dependence. The other is the length change of PMMA-based fiber induced by the moisture absorption. Since the fiber cladding, which is usually made of PMMA, forms the largest portion of the fiber's volume or weight, the fiber length change can be dominated and approximated by that of pure PMMA, even if the fiber core is doped.

The volumetric change induced by water uptake in PMMA can be estimated. Considering a unit volume of an initially dry polymer, of density $\rho_{0}$, which takes up $w$ wt. \% water, the density change of PMMA against water uptake rate can be calculated as [] $]$

$$
\rho=\rho_{0} \frac{1+\frac{w}{100}}{1+\frac{\rho_{u} f w}{100}},
$$

where $f$ is the fraction of the water that contributes to an increase in the PMMA volume and $\rho_{w}$ the density of water. The water absorption varies in different materials. The water absorption can be expressed as [10] 


$$
w=\frac{w_{1} H\left(1-6 H^{5}+5 H^{6}\right)}{(1-H)\left(1-H^{6}\right)},
$$

where $w_{1}$ is the weight of absorbate per gram of adsorbent, which is different for different materials, and for PMMA (Perspex) is 6.25 [10].

The water absorption increases with relative humidity in a nonlinear way defined by Eq. (3). The maximum water absorption can vary for different PMMA fibers from $1.6 \%$ to $2.0 \%$ of total PMMA weight $[\underline{6}, \underline{7}, \underline{10}]$. The volume change of PMMA caused by humidity can be estimated by using Eqs. (2) and (3), and the related swelling coefficient along the fiber direction can be expressed as

$$
\beta=\frac{\Delta V}{3 V_{o}}=\frac{\rho_{u} f w}{300} .
$$

The RI of PMMA varies with relative humidity as well. It was estimated in [7] that the RI of d-PMMA increases as humidity increases at a rate of $1.13 \times 10^{-5}(\% \mathrm{RH})^{-1}$ at $25^{\circ}$ C. This RI humidity dependence decreases as temperature increases. However, the water absorption is a function of relative humidity, $H$, only, and is independent of temperature in experiments [10].

Experiments were carried out to study the humidity response of a POFBG. POFBGs were fabricated by attaching a $10 \mathrm{~cm}$ length of POF to a single mode silica fiber down-lead using UV curable glue (Norland 76). The PMMA-based POF contained a $5 \mathrm{~mm}$ long FBG, fabricated by illuminating from above a phase mask placed on top of the POF using $325 \mathrm{~nm}$ UV light from a $\mathrm{HeCd}$ laser. In the experiments, the POFBGs were placed inside an environmental chamber (Sanyo Gallenkamp) so as to operate at the desired temperature and humidity. They were illuminated via a fiber circulator with light from a broadband light source (Thorlab ASE730) and observed in reflection using an IBSEN I-MON 400 wavelength interrogation system, as shown in Fig. 1.

According to the above analysis of PMMA, one may estimate the wavelength change of a PMMA-based optical fiber grating induced by relative humidity. A calculated result is shown in Fig. 2, where it is assumed that the grating operates at $25^{\circ} \mathrm{C}$, the fraction factor, $f=0.5$, the density of water, $\rho_{w}=0.997$; the effective RI of the fiber core

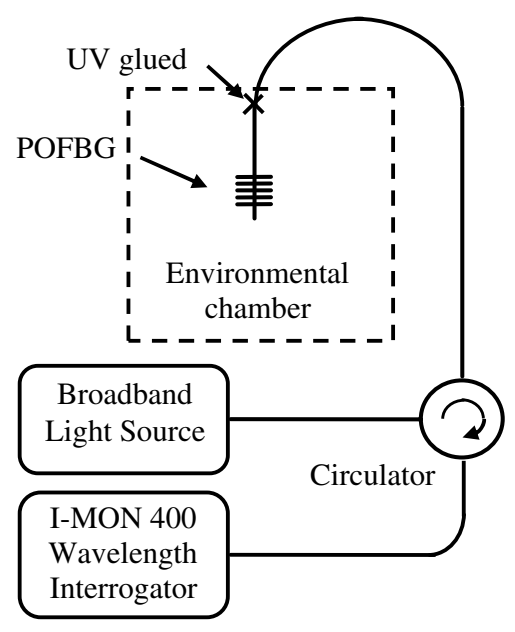

Fig. 1. Experimental arrangement.

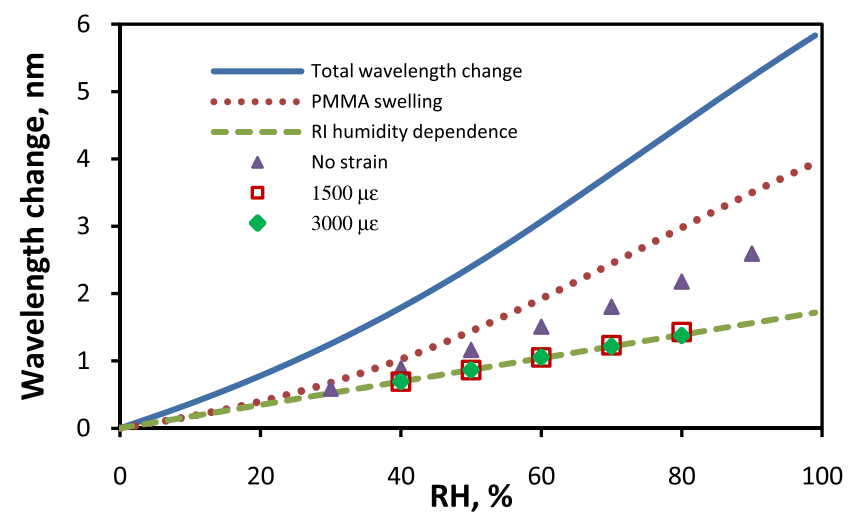

Fig. 2. Calculated and measured wavelength change of POFBG versus RH.

is 1.4825 , which is estimated according to the Bragg wavelength and the phase mask period used in the experiments reported later. At 50\% relative humidity and $25^{\circ} \mathrm{C}$ the estimated swelling coefficient of PMMA, $\beta$, is $3 \times 10^{-5}(\% \mathrm{RH})^{-1}$, larger than the RI change rate, $\eta$, of $1.13 \times 10^{-5}(\% \mathrm{RH})^{-1}$. Therefore, the swelling of PMMA makes the main contribution to the Bragg wavelength change of a POFBG. However, the swelling coefficient is humidity dependent, as defined by Eq. (4).

The environmental chamber was programmed to change the relative humidity from $30 \%$ to $90 \%$ with an increment of $10 \%$ while the temperature was fixed at $25^{\circ} \mathrm{C}$. The average responsivity over $30 \%$ to $90 \%$ of $\mathrm{RH}$ is $34 \pm 3 \mathrm{pm} / \% \mathrm{RH}$. However, the calculated wavelength change shows a much larger responsivity of $66 \mathrm{pm} / \% \mathrm{RH}$ over the same $30 \%$ to $90 \%$ humidity range.

The measured wavelength change is significantly less than that calculated. The difference may arise from several factors. First the PMMA swelling caused by water absorption strongly depends on the fraction factor, $f$, which defines the contribution of water to the increase in PMMA volume. So far the reported $f$ values for PMMA are between 0.4 and 0.6 while the used $f$ value in the calculation is 0.5 , but this factor cannot account for this large difference between the measured and calculated results. Second, the sensitivity of POF may be poorly approximated by that of PMMA, especially the contribution to the humidity dependence of RI arising from the fiber core, which is doped. Finally it has been reported [11] that solid drawn polymers and stretched elastomers exhibit anisotropic expansion, which mainly depends on the polymer processing history. This anisotropy has also been found in PMMA-based optical fibers [12] and implies that linear expansion is not simply a third of volume expansion, with the expansion coefficient believed to be lower in the direction of the molecular orientation than in the direction perpendicular to the draw direction. This may be the major factor accounting for the measured grating response being smaller than expected.

An experiment was designed to further examine the performance of the POFBG. The two ends of a POFBG were clamped to two mounting brackets. One of the brackets was fixed while the other was mounted on a translation stage, which was used to apply a certain strain to the POFBG. The clamped POFBG was then placed in the environmental chamber to test the humidity 
responsivity. Since the ends of the POFBG were clamped the length of the PMMA optical fiber between the two clamping points does not vary with PMMA swelling (given that the applied strain was larger than any humidity induced fiber swelling). In this case, the POFBG humidity responsivity only relies on the RI humidity dependence of the fiber. This is a technique similar to that used in silica fiber Bragg grating strain sensors to remove temperature cross sensitivity [13]. By taking the difference of the humidity responsivities of strained and unstrained POFBGs, one can get the contribution of fiber swelling to the responsivity and find out if anisotropic expansion exists. At $25^{\circ} \mathrm{C}$ the measured wavelength responses of a POFBG under no strain, $1500 \mu \varepsilon$ and $3000 \mu \varepsilon$ were recorded, and their relative wavelength changes are plotted in Fig. 2, together with the calculated wavelength change based on PMMA performance.

The behavior of the POFBG under strain shows that when the POFBG is under a strain larger than the elongation of the fiber due to swelling, the humidity induced fiber swelling makes no contribution to the wavelength change of the POFBG. At this temperature, the RI varies linearly with humidity change. For the POFBG used, the contribution of RI humidity dependence is very close to that calculated by using typical PMMA parameters. This may be a case where the material constants of the fiber core and cladding are well matched. The difference between POFBG responses with and without strain represents the contribution of fiber swelling. As mentioned before, the maximum water absorption varies with different PMMAs from $1.6 \%$ to $2 \%$ in weight. In spite of this variation, the contribution of water swelling from the measured results is much smaller than that predicted. This indicates a reduced swelling coefficient in the POFBG, which we attribute to anisotropic expansion of the fiber.

In the polymer fiber drawing process, the polymer matrix undergoes a chain stretching along the fiber axis. This will orient the polymer molecules along the drawing (axial) direction. This gives rise to optical anisotropy of the fiber in the form of transverse birefringence [12] and mechanical anisotropy in the form of volume expansion [14]. In POFBGs this anisotropy can be mitigated by preannealing the fiber before grating inscription [15]. Even after the annealing of polymer materials, though, there is still a certain amount of anisotropy remaining. The stressrelated anisotropy may vary with environmental conditions, leading to varying responsivity of a POFBG sensor. Pre-straining the POFBG can get rid of this uncertainty at the cost of reduced responsivity. In contrast, an increased responsivity in temperature sensing can be achieved by using a pre-strained POFBG [16].

PMMA-based optical fibers drawn under different conditions will exhibit differing degrees of anisotropic expansion [12]. A further experiment was carried out using a POFBG made in a POF sample similar to the first but fabricated on a different occasion. Since the prestrain applied at different temperatures needs to be larger than the fiber elongation induced by both swelling and thermal expansion $\left(3000 \mu \varepsilon\right.$ for $25^{\circ} \mathrm{C}, 5000 \mu \varepsilon$ for $35^{\circ} \mathrm{C}$, and $8000 \mu \varepsilon$ for $50^{\circ} \mathrm{C}$ in the experiments), the initial POFBG wavelength for each test was very different. Figure $\underline{3}$ shows the measured wavelength change of

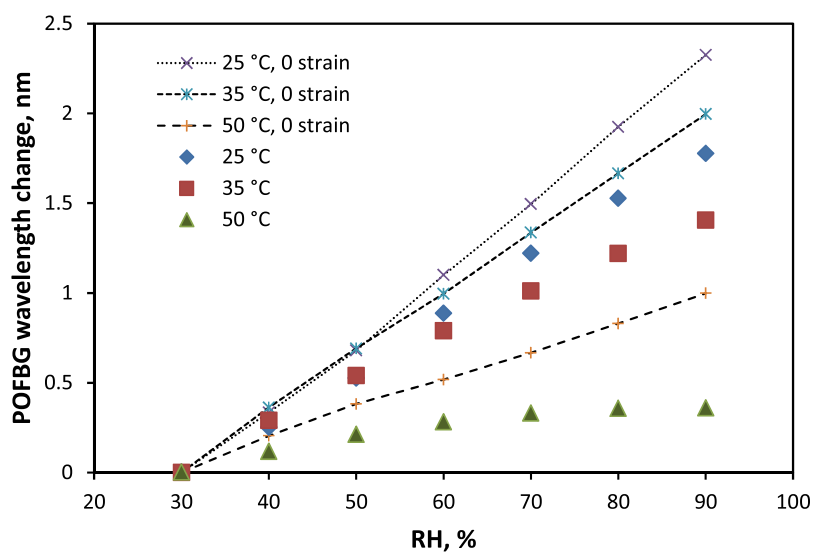

Fig. 3. Measured humidity responses of POFBG with and without pre-strain under different temperatures.

the second POFBG with and without pre-strain at different temperatures. The wavelength change is related to the POFBG wavelength at 30\% RH at each temperature. The unstrained POFBG shows a humidity responsivity of $39 \mathrm{pm} / \% \mathrm{RH}$ at $25^{\circ} \mathrm{C}, 33 \mathrm{pm} / \% \mathrm{RH}$ at $35^{\circ} \mathrm{C}$, and $16 \mathrm{pm} / \% \mathrm{RH}$ at $50^{\circ} \mathrm{C}$. The pre-strained POFBG shows a reduced humidity responsivity of $30 \mathrm{pm} / \% \mathrm{RH}$ at $25^{\circ} \mathrm{C}$, $23 \mathrm{pm} / \% \mathrm{RH}$ at $35^{\circ} \mathrm{C}$, and $6 \mathrm{pm} / \% \mathrm{RH}$ at $50^{\circ} \mathrm{C}$ (where clear nonlinearity is shown). Compared with the result in Fig. 2 the humidity responsivity of this POFBG is larger, with or without pre-strain applied.

For humidity sensing, the POFBG's response time is of interest. For a given type of POF, the response time of a POFBG depends on the diameter of the fiber [17]. Figure 4(a) shows the normalized wavelength changes of the POFBG (with a fiber diameter of $200 \mu \mathrm{m}$ ) over a $10 \%$ step humidity change at $35^{\circ} \mathrm{C}$. The pre-strained POFBG shows a response time of $13 \mathrm{~min}$ at $5000 \mu \varepsilon$ and $8000 \mu \varepsilon$ while the unstrained POFBG exhibits a longer response time of $25 \mathrm{~min}$. Figure 4(b) shows the responses of the POFBG over a $10 \%$ step humidity change at $25^{\circ} \mathrm{C}, 35^{\circ} \mathrm{C}$, and $50^{\circ} \mathrm{C}$ with a pre-strain of $8000 \mu \varepsilon$. The POFBG shows a response time of $19 \mathrm{~min}$ at $25^{\circ} \mathrm{C}, 13 \mathrm{~min}$ at $35^{\circ} \mathrm{C}$, and $11 \mathrm{~min}$ at $50^{\circ} \mathrm{C}$. It indicates that the fiber volume change induced by water swelling takes a longer time to reach equilibrium than the RI change and so pre-straining a POFBG can improve the humidity response time; furthermore, the equilibrium can be established sooner at higher temperature.

The RI humidity dependence of POF can be defined by the Lorentz-Lorenz relation [2,7, and 16]. The RI humidity dependence in a POFBG humidity sensor depends on the changes of the polarizability of the fiber material and fiber volume induced by water content. In a pre-strained POFBG, the expansion in fiber length is restricted, generating a reduced volume change. This generally leads to an increased RI humidity dependence, which could be quantified with the knowledge of the anisotropic volume expansion and the performance of the core material in the POF.

To conclude, the humidity responses of PMMA-based POFBGs have been measured and analyzed in this work. The wavelength change of the POFBG humidity sensor consists of the contributions from the fiber swelling and RI change induced by relative humidity. The analysis 


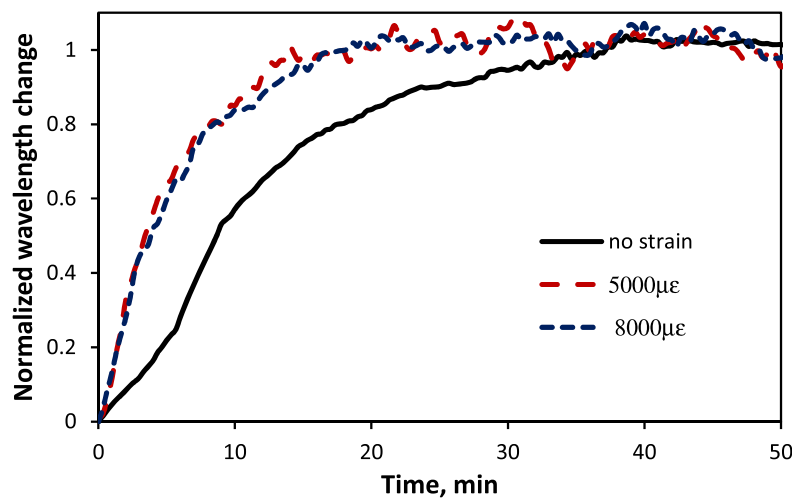

(a)

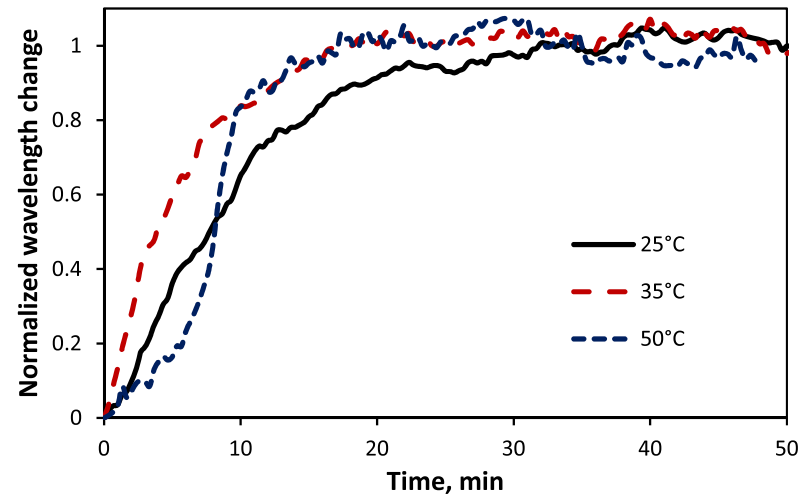

(b)

Fig. 4. Measured responses to humidity change for a POFBG. (a) Under different strains at $35^{\circ} \mathrm{C}$ and (b) at different temperatures with a pre-strain of $8000 \mu \varepsilon$.

shows that there exists anisotropic expansion that reduces the fiber swelling in the fiber length direction and varies with fiber drawing conditions and surrounding environmental conditions. Experiments demonstrate that this uncertainty can be eliminated by pre-straining a POFBG. The POFBG response time is also improved by this technique.
The research leading to these results has received funding from the European Union Seventh Framework Programme (FP7/2007-2013) under grant agreement no. 314032 .

\section{References}

1. C. Zhang, W. Zhang, D. J. Webb, and G.-D. Peng, Electron. Lett. 46, 643 (2010).

2. N. G. Harbach, "Fiber Bragg gratings in polymer optical fibers," Ph.D. dissertation (Ecole Polytechnique Fédérale de Lausanne, 2008).

3. W. Zhang, D. J. Webb, and G.-D. Peng, Opt. Lett. 37, 1370 (2012).

4. C. Zhang, X. Chen, D. J. Webb, and G.-D. Peng, Proc. SPIE 7503, 750380 (2009).

5. G. N. Harbach, H. G. Limberger, and R. P. Salathé, in Proceedings of Bragg Gratings, Photosensitivity, and Poling in Glass Waveguides (Optical Society of America, 2010), paper BTuB2.

6. D. T. Turner, Polymer 23, 197 (1982).

7. T. Watanabe, N. Ooba, Y. Hida, and M. Hikita, Appl. Phys. Lett. 72, 1533 (1998).

8. W. Yuan, L. Khan, D. J. Webb, K. Kalli, H. K. Rasmussen, A. Stefani, and O. Bang, Opt. Express 19, 19731 (2011).

9. G. D. Peng, Z. Xiong, and P. L. Chu, Opt. Fiber Technol. 5, 242 (1999).

10. A. M. Thomas, J. Appl. Chem. 1, 141 (1951).

11. D. R. Salem, Structure Formation in Polymeric Fibers (Hanser-Gardner, 2001).

12. P. Ji, A. D. Q. Li, and G.-D. Peng, Proc. SPIE 5212, 108 (2003)

13. http://www.anibertech.com/Athermal\%20Strain\%20Sensor \%20ASS-01.html.

14. L.-H. Wang, C. L. Choy, and R. S. Porter, J. Polymer Sci. 21, 657 (1983).

15. K. E. Carroll, C. Zhang, D. J. Webb, K. Kalli, A. Argyros, and M. C. J. Large, Opt. Express 15, 8844 (2007).

16. W. Zhang and D. J. Webb, "Factors influencing the temperature sensitivity of PMMA based optical fiber Bragg gratings," in Photonics Europe, Brussels, Belgium, 2014.

17. W. Zhang, D. J. Webb, and G.-D. Peng, J. Lightwave Technol. 30, 1090 (2012). 